\title{
INFRARED ABSORPTION INVESTIGATIONS CONFIRM THE EXTRATERRESTRIAL ORIGIN OF CARBONADO DIAMONDS
}

\author{
Jozsef Garai ${ }^{1}$ and Stephen E. Haggerty \\ Department of Earth Sciences, Florida International University, Miami, FL \\ AND \\ SANDEEP ReKhi ${ }^{2}$ AND MARK Chance \\ Case Western Reserve University, Cleveland, OH; and Case Center for Synchrotron Biosciences, National Synchrotron Light Source, \\ Brookhaven National Laboratory, Upton, NY \\ Received 2006 July 25; accepted 2006 October 27; published 2006 December 7
}

\begin{abstract}
The first complete infrared FTIR absorption spectra of carbonado diamond confirm the interstellar origin of this most enigmatic diamond. All previous attempts failed to measure the absorption of carbonado diamond in the most important IR range of $1000-1300 \mathrm{~cm}^{-1}(10.00-7.69 \mu \mathrm{m})$ because of silica inclusions. In our investigation, $\mathrm{KBr}$ pellets were made from crushed silica-free carbonado diamond, and thin sections were also prepared. The 100-1000 times brighter synchrotron infrared radiation permits a greater spatial resolution. Inclusions and pore spaces were avoided and/or sources of chemical contamination were removed. The FTIR spectra of carbonado diamond mostly depict the presence of single nitrogen impurities and hydrogen. The lack of identifiable nitrogen aggregates in the infrared spectra, the presence of features related to hydrocarbon stretch bonds, and the resemblance of the spectra to CVD and presolar diamonds indicate that carbonado diamonds formed in a hydrogenrich interstellar environment. This is consistent with carbonado diamond being sintered and porous, with extremely reduced metals, metal alloys, carbides, and nitrides, light carbon isotopes, surfaces with glassy melt-like patinas, deformation lamellae, and a complete absence of primary, terrestrial mineral inclusions. The 2.6-3.8 billion year old fragmented body was of asteroidal proportions.
\end{abstract}

Subject headings: ISM: evolution — ISM: general — meteors, meteoroids — methods: laboratory — stars: carbon - techniques: spectroscopic

Online material: color figures

\section{INTRODUCTION}

Carbon is the most abundant dust-forming element in the interstellar medium. The dominant fraction of carbon is in the form of grains, composed principally of hydrocarbons. Other components include graphite, hydrogenated amorphous aliphatic and/or aromatic hydrocarbons $(\mathrm{a}-\mathrm{C}, \mathrm{a}-\mathrm{C}: \mathrm{H})$, and diamond. The existence of interstellar diamond was predicted by Saslaw \& Gaustad (1969) and subsequently found in primitive, carbonaceous chondritic meteorites (Lewis et al. 1987). Interstellar diamonds are cubic and coexist with highly reduced carbides of $\mathrm{Si}, \mathrm{Mo}, \mathrm{W}$, and Ti. Although there is widespread consensus that diamonds can form in interstellar environments (Kouchi et al. 2005; Binette et al. 2005), the processes are debated (Lewis et al. 1987; Tielens et al. 1987; Daulton et al. 1995; Nuth \& Allen 1992; Duley \& Grishko 2001).

The presence of diamond-related bands at 2915 and $2832 \mathrm{~cm}^{-1}$ (3.43 and $3.53 \mu \mathrm{m}$ ) in the IR emission spectra of two Herbig Ae/Be stars, HD 97048 and Elias 1 (Guillois et al. 1999; Van Kerckhoven et al. 2002), is well established. A tentative peak at $2832 \mathrm{~cm}^{-1}(3.53 \mu \mathrm{m})$ has also been detected in V921 Sco (=CD 42.11721), HD 163296, and T CrA (Acke \& Van den Ancker 2006). The bands are interpreted as surface $\mathrm{C}-\mathrm{H}$ stretching modes of hydrogenated nanodiamonds, with particle sizes in HD 97048 and Elias 1 of at least $50 \mathrm{~nm}$ (Jones et al. 2004).

Terrestrial diamonds are recovered from exotic volcanoes (kimberlites and lamproites) that originate at depths $>150 \mathrm{~km}$. The single exception is carbonado, a tough, black to dark gray polycrystalline cuboid diamond (Fig. 1), 2.6-3.8 Gyr in age,

\footnotetext{
${ }^{1}$ Corresponding author; jozsef.garai@ fiu.edu.

${ }^{2}$ Also at Physics Department, Harvard University, Cambridge, MA.
}

found in the same sedimentary geological horizon in Brazil and the Central African Republic (Haggerty 1998; Heaney et al. 2005 and references therein). Surfaces are commonly patinated, and samples are coke or ceramic-like (Haggerty 1998) with vesicles of up to $1 \mathrm{~mm}$ (Fig. 1) and porosities of up to $\sim 30 \%$ (Vicenzi et al. 2003); the C isotopic composition $\left(\delta^{13} \mathrm{C} \%\right.$ o is between -21 and -34 ) is extremely light (Russell et al. 1996; Shelkov et al. 1997); planar-defect shock lamellae are present (Heaney et al. 2005); and the diamonds contain an extraordinary array of highly reduced metals and metal alloys (Fe, Ni, Fe-Ni, Fe-Cr, Ni-Cr), SiC, and TiN (De \& Heaney 1996; Gorshkov et al. 1996; Jones et al. 2002; Parthasarathy et al. 2004). Because none of the above characteristics are compatible with a deep Earth environment, a reasonable alternative is that carbonado diamond is of extraterrestrial origin (Haggerty 1996, 1998, 1999). This proposal is furthered explored here in the context of the IR spectra of carbonado.

An important physical characteristic of diamond, which may shed light on its origin, is the aggregation level, as outlined in $\S 3.2$, of substituted nitrogen (Table 1). The IR absorption in the most important nitrogen-related region, between 1000 and $1300 \mathrm{~cm}^{-1}(10.00-7.69 \mu \mathrm{m})$, has never been collected from carbonado diamond. The pores of carbonado are typically stuffed with silica-based minerals that yield a broad absorption feature between 900 and $1300 \mathrm{~cm}^{-1}(11.11$ and $7.69 \mu \mathrm{m})$. The intensity of this feature is much stronger than the defect-activated single-phonon bands, thus preventing the measurement of the infrared absorption of carbonado diamond (Kagi et al. 1994; Magee \& Taylor 1999). No experiments have succeeded in overcoming these problems, and the diamond type (e.g., Ia, $\mathrm{IaA}, \mathrm{IaB}, \mathrm{II})$ in carbonado has yet to be determined. 


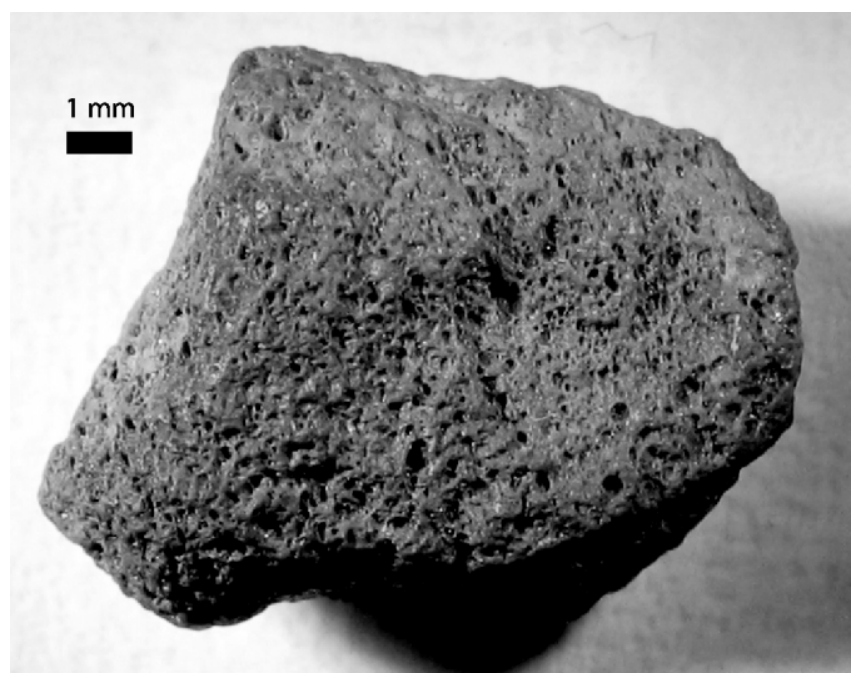

FIG. 1.-Typical black and highly porous polycrystalline carbonado diamond (5.3 carats) from Lencois, Bahia, Brazil (sample B-13). [See the electronic edition of the Journal for a color version of this figure.]

Electron paramagnetic resonance of carbonado diamond shows the presence of single substitutional nitrogen centers, labeled P1 (Smith et al. 1959; Braatz et al. 2000; Nadolinny et al. 2003). Defects containing hydrogen, labeled H1, and higher $\mathrm{N}$ aggregation centers, $\mathrm{N}_{2}^{+}, \mathrm{N}_{2} \mathrm{~V}^{+}$, and $\mathrm{N}_{3} \mathrm{~V}$, reported by Nadolinny et al. (2003), are, however, not observed by others (e.g., Braatz et al. 2000).

\section{EXPERIMENTAL}

In order to remove silica contamination from carbonado diamonds, two Brazilian and two Central African samples (3-5 g) were crushed in a tungsten carbide piston cylinder crusher to $<67 \mu \mathrm{m}$. The resulting powders were treated with $\mathrm{HCl}$ for 3 days and then placed in $\mathrm{HF}$ pressure bombs at $60^{\circ} \mathrm{C}$ for an additional 3 days. The thoroughly washed, recovered carbonado diamond was mixed with $\mathrm{KBr}$ in different proportions and then pressed under low vacuum to form $\sim 1 \mathrm{~mm}$ thick pellets. A control pellet with the same weight proportion was made from natural diamond crushed to $\sim 25 \mu \mathrm{m}$. Three thin sections of carbonado diamond, two from the Central African Republic and one from Brazil, were also prepared with no chemical treatment.

The synchrotron IR experiment was carried out at the U2b beam line located at the National Synchrotron Light Source at Brookhaven National Laboratory. The beam line is equipped with a dry-nitrogen-purged FTIR spectrometer (Nicolet Magna $860)$ with a $\mathrm{KBr}$ beam splitter and a narrowband mercury cadmium telluride (MCT/A) detector coupled with an IR microscope (Nic-Plan, Nicolet Instruments, Madison, Wisconsin) with 32X IR objective (Thermo Spectra-Tech) and diffraction-limited resolution. The spectrometer is equipped with a Michelson interferometer having capabilities such as rapid scan and step scan, and the ability to accept external beams and detectors. The bench is configured to use a collimated synchrotron light (beam energy $800 \mathrm{MeV}$ ) through an external input of the spectrometer. The IR spectra of various carbonado samples mixed with dry $\mathrm{KBr}$ were collected with a beam spot diameter around $100 \mu \mathrm{m}$. The background spectra (1064 scans) were collected and subtracted from the IR spectra of the samples. The IR spectra were collected in the mid-IR range $4000-650 \mathrm{~cm}^{-1}(2.50-15.34 \mu \mathrm{m})$ at a resolution of $4 \mathrm{~cm}^{-1}$ with 1056 scans co-added. Stage control, data collection, and processing were performed using OMNIC 7.2 (Thermo-Nicolet, Madison, Wisconsin). The portions of carbon-
TABLE 1

Commonly Observed Bands IN THe IR Spectrum OF DIAMOND

\begin{tabular}{|c|c|}
\hline $\begin{array}{l}\text { Frequency } \\
\quad\left(\mathrm{cm}^{-1}\right)\end{array}$ & Functional Group \\
\hline $1130(8.85 \mu \mathrm{m}) \ldots$ & Substitutional N \\
\hline $1175(8.51 \mu \mathrm{m}) \ldots \ldots \ldots$ & B aggregate \\
\hline $1220(8.20 \mu \mathrm{m}) \ldots$ & $\mathrm{C}-\mathrm{N}$ stretch \\
\hline $1250(8.00 \mu \mathrm{m}) \ldots \ldots \ldots$ & $\mathrm{C}-\mathrm{N}, \mathrm{N}-\mathrm{V}$ \\
\hline $1282(7.80 \mu \mathrm{m}) \ldots \ldots \ldots$ & A aggregates (pairs) \\
\hline $1332(7.51 \mu \mathrm{m}) \ldots \ldots \ldots$ & Raman \\
\hline $1350(7.41 \mu \mathrm{m}) \ldots \ldots \ldots$ & Substitutional N \\
\hline$\sim 1370(\sim 7.30 \mu \mathrm{m}) \ldots \ldots$ & Platelet N \\
\hline $1405(7.12 \mu \mathrm{m}) \ldots \ldots \ldots$ & Overtone \\
\hline
\end{tabular}

Note. - The frequency of the Raman group is intrinsic. The other frequencies are from McNamara (2003) and references therein.

ado samples were scanned and selected using a CCD camera linked to the infrared images (objective 32X).

\section{RESULTS AND DISCUSSION}

Using a glow bar (blackbody) generated source, the infrared absorption spectra of the pellets were collected from 650 to 4000 $\mathrm{cm}^{-1}(15.34-2.50 \mu \mathrm{m})$. Outside the nitrogen absorption region $1000-1500 \mathrm{~cm}^{-1}$, additional bands in the $650-1000 \mathrm{~cm}^{-1}(15.34$ $6.67 \mu \mathrm{m})$ region and a triplet around $2900 \mathrm{~cm}^{-1}(3.45 \mu \mathrm{m})$ were observed in the carbonado diamond spectra. The additional nonnitrogen absorption peaks are interpreted as $\mathrm{C}-\mathrm{H}$ related bands.

Using a synchrotron radiation source with a 100 times better signal-to-noise ratio than the laboratory instrument using a glow bar infrared source (Reffner et al. 1994), the absorption spectra of the thin sections were collected from 650 to 4000 $\mathrm{cm}^{-1}(15.34-2.50 \mu \mathrm{m})$. The prominent peaks present in all spectra are 1102, 2855, 2926, and $2961 \mathrm{~cm}^{-1}(9.07,3.50,3.42$, and $3.38 \mu \mathrm{m})($ Fig. 2; Table 2). Additional sharp peaks are also present but are not developed in all runs. There are relatively broad features, present in the three thin section investigations, at $1660,2030,2155 \mathrm{~cm}^{-1}(6.02,4.93,4.64 \mu \mathrm{m})$.

Any possibility of contamination from the instrument and/or contamination by organic solvents was ruled out by taking the spectra of natural diamond, which did not show $\mathrm{C}-\mathrm{H}$ related peaks.

\subsection{C-H Stretching Bands}

The peak frequencies (Fig. 2) at 2855, 2926, and $2961 \mathrm{~cm}^{-1}$ (3.50, 3.42, and $3.38 \mu \mathrm{m})$ can be attributed to $\mathrm{C}-\mathrm{H}$ stretching of diamond hydride and are sensitive to the surface structure (Chin et al. 1995; Alfonso et al. 1995; Zhigilei et al. 1997). Hydrogen ionization experiments on diamond single crystals show a band at $2835 \mathrm{~cm}^{-1}(3.53 \mu \mathrm{m})$ that is ascribed to $\mathrm{C}(111)-1 \times 1: \mathrm{H}$ [i.e., single carbon-hydrogen bonds on octahedral (111) crystallographic faces], a doublet at 2921 and $2935 \mathrm{~cm}^{-1}$ (3.42 and $\left.3.41 \mu \mathrm{m}\right)$ associated with $\mathrm{C}-\mathrm{H}$ on (100), and 2857 and $2874 \mathrm{~cm}^{-1}$ ( 3.50 and $3.48 \mu \mathrm{m}$ ) bands related to (110) (Chin et al. 1995; Cheng et al. 1996). The complete assignment of $\mathrm{C}-\mathrm{H}$ stretching in polycrystalline diamonds, however, is incomplete.

The (111) surface peak is absent in our spectra. The prominent bands at 2855 and $2926 \mathrm{~cm}^{-1}(3.50$ and $3.42 \mu \mathrm{m})$ are assigned respectively to $\mathrm{CH}_{x}$ and $\mathrm{C}\{100\}$ (Guillois et al. 1999; Jones et al. 2004). The band observed at $2961 \mathrm{~cm}^{-1}(3.38 \mu \mathrm{m})$ and reported in other studies remains unassigned. The lack of the $\mathrm{C}(111)-1 \times 1: \mathrm{H}$ surface, but the presence of the $\mathrm{C}\{100\}$ surface in the carbonado diamond spectra, is fully consistent 


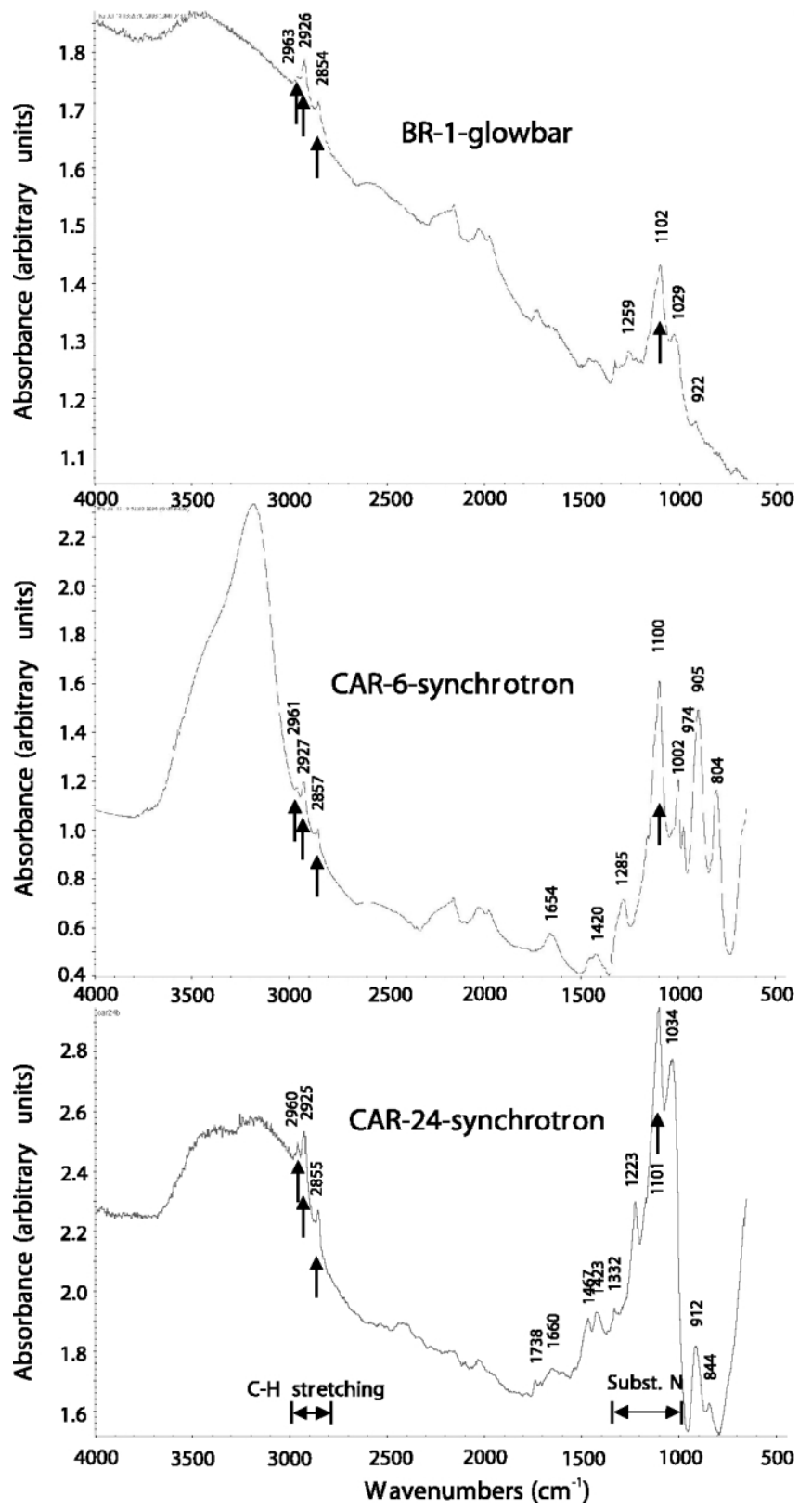

FIG. 2.-Mid-infrared absorption spectra of carbonado diamonds collected from Central African (CAR) and Brazilian (BR) thin sections. The prominent peaks are marked with arrows, and the assigned vibrational regions are identified. [See the electronic edition of the Journal for a color version of this figure.]

with the prevalent cuboid habit of individual diamond crystals in our carbonado collection. And it is consistent with "asteriated" (zoned and star-shaped) diamonds in which cuboidal forms have a strong preference for hydrogen, and octahedral segments for nitrogen (Rondeau et al. 2004). More generally, hydrogen is critical to diamond formation in CVD processes, is present as "dangling bonds" (i.e., singly occupied, outwardly directed $s p^{3}$ hybrid orbitals associated with each surface carbon atom) in natural diamonds (Evans 1992), and is invoked in "hydrogenated nanodiamonds" in meteorites (Andersen et al. 1998) and with "protonation" as the bonding agent that sinters microdiamonds in carbonado (Haggerty 1998).

\subsection{Nitrogen-related Bands}

Nitrogen $(Z=7)$ may replace carbon $(Z=6)$, but long-term residence at high temperatures results in $\mathrm{N}$ migration and high
TABLE 2

The Assignment of the Prominent Bands Observed in the Carbonado Diamond FTIR Spectra

\begin{tabular}{|c|c|c|c|}
\hline $\begin{array}{l}\text { Frequency } \\
\left(\mathrm{cm}^{-1}\right)\end{array}$ & Assignment & Intensity & $\begin{array}{c}\text { FWHM } \\
(\mu \mathrm{m})\end{array}$ \\
\hline $1102(9.07 \mu \mathrm{m})$ & $\mathrm{C}-\mathrm{N} / \mathrm{C}-\mathrm{O}$ stretch & 1.00 & 0.347 \\
\hline $2855(3.50 \mu \mathrm{m})$ & $\mathrm{CH}_{x}$ & 0.08 & 0.027 \\
\hline $2926(3.42 \mu \mathrm{m}) \ldots \ldots$ & $C\{100\}$ & 0.18 & 0.029 \\
\hline $2961(3.38 \mu \mathrm{m}) \ldots \ldots$ & $\ldots$ & 0.11 & 0.038 \\
\hline
\end{tabular}

aggregation levels (Evans \& Qi 1982); short-term residence at low temperatures, or quenched CVD diamonds, corresponds primarily to single $\mathrm{N}$ as a substitutional impurity. We observe a prominent absorption peak (present in all of the spectra) in the $\mathrm{C}-\mathrm{N}$ stretching region at $1102 \mathrm{~cm}^{-1}(9.07 \mu \mathrm{m})$, which is also reported in the IR spectra of presolar diamonds from the Allende meteorite (Lewis et al. 1989) and in CVD diamonds (Andersen et al. 1998). The peak is attributed to $\mathrm{C}-\mathrm{N}$ stretching and/or $\mathrm{C}-\mathrm{O}$ stretching in aliphatic ethers (Koike et al. 1995; Colangeli et al. 1994; Mutschke et al. 1995; Hill et al. 1997).

The peak at $1102 \mathrm{~cm}^{-1}(9.07 \mu \mathrm{m})$ is relatively wide (FWHM $=103.6 \mathrm{~cm}^{-1}$ ) and asymmetric with a shoulder, indicative of two peaks. Spectral analyses show peaks at $1100 \mathrm{~cm}^{-1}$ $(9.09 \mu \mathrm{m})$ and $1128 \mathrm{~cm}^{-1}(8.86 \mu \mathrm{m})$ that reproduce the observed spectra. The $1128 \mathrm{~cm}^{-1}$ is attributed to substitutional nitrogen. The presence of a single substitutional nitrogen $[\mathrm{C}-\mathrm{N}]^{\circ}$, or $\mathrm{P} 1$, center in the carbonado diamond is well established from electron paramagnetic resonance investigations (Smith et al. 1959; Braatz et al. 2000; Nadolinny et al. 2003), adding additional support to the existence of this peak. Hydrogen may have an effect on peak shifts, and the influence of deformation or radiation has not been fully explored.

In the spectra of CAR-24, which has the most intensive 1102 $\mathrm{cm}^{-1}$ peak, the accompanying asymmetric $\mathrm{C}-\mathrm{O}-\mathrm{C}$ stretch peak at $1223 \mathrm{~cm}^{-1}(8.18 \mu \mathrm{m})$ is present, supporting the $\mathrm{C}-$ $\mathrm{O}-\mathrm{C}$ origin for the peak. On the other hand, the possible nitrogen attribution of this band is supported by the increasing intensity of the band with nitrogen doping (Titus et al. 2006).

The other substitutional-nitrogen-related peak at $1350 \mathrm{~cm}^{-1}$ $(7.41 \mu \mathrm{m})$ is not present in our spectra, which is consistent with its absence in the reported spectra of presolar and CVD diamonds (Andersen et al. 1998). Another, possible nitrogenrelated peak at $1285 \mathrm{~cm}^{-1}(7.78 \mu \mathrm{m})$ is observed only in the spectra of CAR-6; its position, if neither red- nor blueshifted, could be ascribed to A aggregation of $\mathrm{N}$ pairs. Two $\mathrm{N}$ atoms and a vacancy (designated as $3 \mathrm{H}$ and $\mathrm{H} 3$ centers), are typically the result of radiation or deformation and are present in the photoluminescence spectra of carbonado diamond (Clark et al. 1992; Nadolinny et al. 2003). There is no indication of higher order nitrogen centers or of $\mathrm{N}$ platelets.

The absorption peak at $1384 \mathrm{~cm}^{-1}(7.22 \mu \mathrm{m})$ reported by Kagi et al. (1994) has not been confirmed by other investigators. The position of the platelet absorption peak is correlated with nitrogen abundance and inversely with platelet size; therefore, the $1384 \mathrm{~cm}^{-1}(7.22 \mu \mathrm{m})$ absorption peak should be due to small platelets sizes and with abundant nitrogen. Carbonado diamond, however, has low nitrogen contents (100-300 ppm) that should result in large platelets and a corresponding redshift, rather than the blueshift suggested by Kagi et al. (1994). The absorption peak of aggregated platelets (at $\sim 1370 \mathrm{~cm}^{-1}[\sim 7.30$ $\mu \mathrm{m}]$ ) is always accompanied by an A absorption peak at 1282 $\mathrm{cm}^{-1}(7.80 \mu \mathrm{m})$ (Davies 1977; Mendelssohn \& Milledge 1995), but is absent in the spectra collected by Kagi et al. (1994). The absence of the A aggregates (pairs) in the spectra raise the 
possibility that the $1384 \mathrm{~cm}^{-1}$ absorption peak might not originate from diamond. One explanation might be that the 1384 $\mathrm{cm}^{-1}$ peak could be due to BN contamination (Mendelssohn \& Milledge 1995) in the stainless container used to crush the carbonado (Kagi et al. 1994).

\subsection{Other Peaks}

The broadband features at 2030 and $2155 \mathrm{~cm}^{-1}$ (4.93 and $4.64 \mu \mathrm{m})$ are most likely related to diamond double-phonon absorptions (Klein et al. 1992). The $1660 \mathrm{~cm}^{-1}(6.02 \mu \mathrm{m})$ band can be assigned to $\mathrm{C}=\mathrm{C}$. The remaining bands with welldeveloped peaks from 800 to $1500 \mathrm{~cm}^{-1}(12.5-6.67 \mu \mathrm{m})$ can be assigned to PAHs (polycyclic aromatic hydrocarbons) (Allamandola et al. 1989). The broad feature in CAR-6 at 3195 $\mathrm{cm}^{-1}(3.13 \mu \mathrm{m})$ can be attributed to $\mathrm{N}-\mathrm{H}$ stretching.

The presence of PAH bands in the spectra is consistent with carbonado diamonds containing $\sim 0.002-0.004$ wt. \% (Kaminsky 1991); hydrocarbon peaks were also obtained from the thin sections, confirming that these are not an artifact of hydrofluoric acid treatment.

\section{CONCLUSIONS}

From the first complete infrared FTIR absorption spectra of carbonado diamond we show that hydrogen stretching bands at 2855,2926 , and $2961 \mathrm{~cm}^{-1}(3.50,3.42$, and $3.38 \mu \mathrm{m})$ are present. The strongest absorption band at $1102 \mathrm{~cm}^{-1}(9.04 \mu \mathrm{m})$ is most likely due to substitutional nitrogen in hydrogenated diamond; however, the possibility of a $\mathrm{C}-\mathrm{O}-\mathrm{C}$ vibration cannot be excluded. All of the prominent peaks are present in treated and untreated samples and are intrinsic to carbonado diamond. These bands may be useful in the identification of extraterrestrial diamond.

The identified absorption peaks, with the exception of some PAH-related bands, are almost identical with the spectra reported for presolar and CVD diamonds (Andersen et al. 1998; Braatz et al. 2000), indicating that these diamonds were most likely formed in similar, hydrogen-rich environments.

Based on the overwhelming evidence for a close resemblance of the FTIR spectra of carbonado diamond to presolar diamonds in meteorites and to interstellar diamond dust; the overlapping range of $\mathrm{C}$ isotopic compositions with meteoritic nanodiamonds; the high porosity; the high concentration of PAHs; the cuboid-shaped microcrystals; exotic metal, metal alloy, SiC, and TiN inclusions (found only in meteorites); the high concentration of planar-defect lamellae; and the complete absence of a deep Earth mantle fingerprint, we conclude that the new IR measurements are consistent with an origin of carbonado diamonds in an interstellar environment. The implication is that carbonado diamond is of asteroidal proportions. This is entirely reasonable given that crystalline white dwarfs are interpreted as modified diamond (Metcalf et al. 2004), and that extrasolar carbon planets are posited as containing an inner, concentric horizon of diamond (Kuchner \& Seager 2005).

Support by the NSF (EAR 95-05770), the Office of Grants and Contracts at the University of Massachusetts, and DeBeers for field work and laboratory studies by S. E. H. is greatly appreciated. Thanks to Jen Bohon (CSB, NIBIB P41-EB01979) for helping in the collection of some IR spectra, and to the reviewers for suggested improvements.

\section{REFERENCES}

Acke, B., \& Van den Ancker, M. E. 2006, A\&A, 457, 171

Alfonso, D. R., Drabold, D. A., \& Ulloa, S. E. 1995, Phys. Rev. B, 51, 1989

Allamandola, L. J., Tielens, A. G. G. M., \& Barker, J. R. 1989, ApJS, 71, 733

Andersen, A. C., et al. 1998, A\&A, 330, 1080

Binette, L., et al. 2005, ApJ, 631, 661

Braatz, A., et al. 2000, Meteoritics Planet. Sci. 35, 75

Cheng, C. L., et al. 1996, J. Chem. Phys., 105, 8977

Chin, R. P., et al. 1995, Phys. Rev. B, 52, 5985

Clark, C. D., Collins, A. T., \& Woods, G. S. 1992, in The Properties of Natural and Synthetic Diamond, ed. J. E. Field (New York: Academic Press), 35

Colangeli, L., et al. 1994, A\&A, 284, 583

Daulton, T. L., et al. 1995, Lunar Planet Sci. Conf., 26, 313

Davies, G. 1977, Chemistry and Physics of Carbon, 13, 1

De, S., \& Heaney, P. J. 1996, Eos, 77, S143

Duley, W. W., \& Grishko, Y. I. 2001, ApJ, 554, L209

Evans, S. 1992, in The Properties of Natural and Synthetic Diamond, ed. J. E. Field (New York: Academia Press), 182

Evans, T., \& Qi, Z. 1982, Proc. R. Soc. London A, 382, 159

Gorshkov, A. I., Titkov, S. V., Pleshakov, A. M., Sivtsov, A. V., \& Bershov, L. V. 1996, Geologiya Rudnykh Mestorozhdenii (Geology of Ore Deposits), 38, 114

Guillois, O., Ledoux, G., \& Reynaud, C. 1999, ApJ, 521, L133

Haggerty, S. E. 1996, Eos, 77, S143 1998, in Advanced Materials '98: Proc. 5th NIRIM Int. Symp. on

Advanced Materials, ed. M. Kamo et al. (Tsukuba: NIRIM), 39 1999, Science, 285, 851

Heaney, P. J., Vicenzi, E. P., \& De, S. 2005, Elements, 1, 85

Hill, H. G. M., et al. 1997, Meteoritics Planet. Sci., 32, 713

Jones, A. P., Milledge, H. J., \& Beard, A. D. 2002, in Poster Abstracts of the Eighth Int. Kimberlite Conf. (Victoria), 3.P24

Jones, A. P., et al. 2004, A\&A, 416, 235
Kagi, H., et al. 1994, Geochim. Cosmochim. Acta, 58, 2629

Kaminsky, F. V. 1991, in Proc. Fifth Int. Kimberlite Conf. (Araxá), 2, 136

Klein, C. A., Hartnett, T. M., \& Robinson, C. J. 1992, Phys. Rev. B, 45, 12854

Koike, C., et al. 1995, MNRAS, 277, 986

Kouchi, A., Nakano, H., Kimura, Y., \& Kaito, C. 2005, ApJ, 626, L129

Kuchner, M. J., \& Seager, S. 2005, preprint (astro-ph/0504214)

Lewis, R. S., Anders, E., \& Draine, B. T. 1989, Nature, 339, 117

Lewis, R. S., et al. 1987, Nature, 326, 160

Magee, C. W., \& Taylor, W. R. 1999, in Proc. 7th Int. Kimberlite Conf. (Cape Town), 529

McNamara, K. M. 2003, Appl. Phys. Lett., 83, 1325

Mendelssohn, M. J., \& Milledge, H. J. 1995, Int. Geology Rev., 37, 95

Metcalf, T. S., Montgomery, M. H., \& Kanaan, A. 2004, ApJ, 605, L133

Mutschke, H., Dorschner, J., Henning, Th., \& Jäger, C. 1995, ApJ, 454, L157

Nadolinny, V. A., et al. 2003, Am. Mineralogist, 88, 11

Nuth, J. A., \& Allen, J. E. 1992, Ap\&SS, 196, 117

Parthasarathy, G., et al. 2004, in India-United States Conference on Space Science, Application, and Commerce (Bangalore: Astronautical Soc. India), 23

Reffner, J., et al. 1994, Synchrotron Radiation News, 7, 30

Rondeau, B., et al. 2004, Diamond \& Related Materials, 13, 1658

Russell, S. S., et al. 1996, Meteoritics Planet. Sci., 31, 343

Saslaw, W. C., \& Gaustad, J. E. 1969, Nature, 221, 160

Shelkov, D., et al. 1997, in Proc. 6th Int. Kimberlite Conf. (Novosibirsk), 38, 322

Smith, W. V., et al. 1959, Phys. Rev., 115, 1546

Tielens, A. G. G. M., et al. 1987, ApJ, 319, L109

Titus, E., et al. 2006, Thin Solid Films, 515, 201

Van Kerckhoven, C., et al. 2002, A\&A, 384, 568

Vicenzi, E. P., et al. 2003, Geochim. Cosmochim. Acta Suppl., 67(18), 513

Zhigilei, L. V., Srivastava, D., \& Garrison, B. 1997, Surface Sci., 374, 333 